\title{
Research Progress in Pathophysiological Mechanism of Dyskinesia in Parkinson's Disease
}

\author{
ZHE ZHONG, MIN XU, LAN QING YANG, W. J. CHEN, HUAN GAO AND MIN YE* \\ Department of Neurology, Affiliated BenQ Hospital of Nanjing Medical University, Nanjing, Jiangsu 210019, China
}

Zhong et al.: Pathophysiological Mechanism of Dyskinesia in Parkinson's Disease

\begin{abstract}
This study involves the research progress in pathophysiological mechanism of dyskinesia in Parkinson's disease. Levodopa-induced dyskinesia is a complication characterized by involuntary movements in Parkinson's patients after long-term treatment with levodopa. Dyskinesia is a kind of abnormal movement involving the face, neck, arm, leg and axial muscles. It often occurs on the serious side of the disease. It may be manifested as variety of phenomenologies, such as choreiform, dystonia, ballism, myoclonus and other forms of abnormal movements can manifest as well. At present, the research on the mechanisms of levodopa-induced dyskinesia is not yet fully understood. Nigrostriatal degeneration and levodopa administration are considered to be the basis of levodopa-induced dyskinesia. There appears to be variety mechanisms involved, such as abnormal dopaminergic transmission in presynaptic and postsynaptic membrane as well as non-dopaminergic systems. Nigrostriatal degeneration is considered to be the basis and premise of levodopa-induced dyskinesia. With the deepening of the research, non-dopaminergic systems such as glutamate, serotonin, adenosine, cholinergic and epinephrine have been proved to be involved in the occurrence of dyskinesia in animal models and clinical studies. This article is a more detailed review of the possible pathophysiological mechanisms of levodopa-induced dyskinesia, especially non-dopaminergic systems.
\end{abstract}

Key words: Parkinson's disease, levodopa-induced dyskinesia, pathophysiology mechanisms, nondopaminergic systems

Levodopa (L-DOPA) has been the "gold standard" for the treatment of Parkinson's disease (PD) since it came out in the 1960s. However, the enthusiasm for the curative effect of L-DOPA has been gradually eroded by the complications caused by long-term administration. Levodopa induced dyskinesia (LID) is a headache type of motor complications, LID is usually divided into "peak dose dyskinesia", "Biphasic dyskinesia" and "off period dystonia"[1]. For clinicians, how to effectively manage dyskinesia is a difficult problem. Only a better understanding of the pathophysiological mechanisms of dyskinesia can lay the foundation for further precise medical treatment. This article reviews the literature about the pathophysiological mechanisms of LID, looks forward to provide reference for daily clinical work. Clinical manifestations of dyskinesia are as follows. Dyskinesia is a kind of abnormal movement involving the face, neck, arm, leg and axial muscles. It often occurs on the serious side of the disease. It may be manifested as variety of phenomenologies, such as choreiform, dystonia, ballism, myoclonus and other forms of abnormal movements can manifest as well. The clinical manifestation of dyskinesia is closely related to the plasma levels of L-DOPA. LID can be classified into three kinds of clinical common types, peak dose dyskinesia: it is the most common type and may occur as typical "head bobbing" and "trunk swaying" movements when the head and neck are involved. Occasionally, accumulating respiratory muscles may cause shortness of breath and irregularity ${ }^{[2]}$. Biphasic dyskinesia: It manifests as a stereotyped repetitive seizure of the unilateral leg with the rise and fall of levels of L-DOPA. Off-period dystonia: it frequently occurs in the early morning or when the effective concentration of the Dopamine (DA) is lowest, with painful foot flexion and inversion. Pathophysiology of dyskinesia is described below. At present, the research

*Address for correspondence

E-mail: yemin197001@sina.com 
on the mechanisms of LID is not yet fully understood. Nigrostriatal degeneration and L-DOPA administration are considered to be the basis of LID. There appears to be variety mechanisms involved, such as abnormal dopaminergic transmission in presynaptic and postsynaptic membrane as well as non-dopaminergic systems. Nigrostriatal degeneration is considered to be the basis and premise of LID. Current studies have shown that healthy control groups and patients with restless legs do not have dyskinesia's after long-term treatment with L-DOPA ${ }^{[3]}$. The degree of degeneration of the substantia nigra striatum is correlated with the severity of involuntary movement, which are more likely to occur on the affected side ${ }^{[4]}$. The nigrostriatal system has a good compensatory ability in the early development of disease. On the one hand, it can increase DA conversion and reduce DA reuptake to compensate for the decrease of DA. On the other hand, DA is stored in presynaptic vesicles to maintain the presynaptic DA concentration and the relatively constant stimulation of postsynaptic receptors ${ }^{[5]}$. As the disease progresses, the storage and conversion capacity of DA in striatum is weakened. The post-synaptic DA receptors are subject to fluctuating stimulation and cause changes in the postsynaptic membrane signaling pathway, leading to the occurrence of LID. Nevertheless, dopaminergic denervation is not sole explanation for the development of LID. L-DOPA administration is another main factor in developing dyskinesia. There is the exact relationship between the L-DOPA administration and the clinical phenotype of dyskinesia ${ }^{[6]}$. Peak does dystonia mostly occurs when the plasma concentration is the highest, biphasic dystonia occurs when L-DOPA just increases and decreases, whereas the off-phase dystonia often occurs when the level is low. In addition, the pharmacokinetics of L-DOPA plays an important role in it. L-DOPA has a short half-life $(1.5 \mathrm{~h})$ and rapidly metabolized in plasma, which makes its blood concentration fluctuate greatly. This method destroys the initially impaired presynaptic buffering capacity, exposing the DA receptors to pulsatile, non-physiologic stimulation, which eventually causes the development of LID. Presynaptic mechanism is as follow. As mentioned above, the L-DOPA is converted into DA in the remaining DA neurons and released through vesicles in the early stage of the disease. Since the remaining neurons can compensate for part of the function, the general striatum (sensory nerve conduction (SNC)) loses at least $50 \%$ of the dopaminergic neurons before the PD motor symptoms appear ${ }^{[7]}$. With the progression of the disease, other cells like endothelial cells, glial cells and 5-HT neurons will compensate decarboxylate L-DOPA into DA, due to lack of a normal physiological regulation mechanism, which will lead to uncontrolled DA release and aggravate DA receptor pulses ${ }^{[8]}$. Postsynaptic mechanism is as follow. It is currently believed that the nigrostriatal degeneration and L-DOPA administration change the function and anatomical structure of the striatum, which cause hypersensitivity to medium spiny neurons (MSN) in the striatum ${ }^{[9]}$. Increasing evidence suggests that spines dynamically change their density and morphology and that these changes are closely related to synaptic plasticity. It is reported that spine head size increase in the induction of long-term potentiation (LTP) and decrease with long-term depression (LTD) ${ }^{[10]}$. Therefore, it is speculated that the change in the head of the spine process may be the structural basis for the occurrence of LID. In addition, the hypersensitivity of dopamine D1 receptor has gradually received more and more attention in the development of LID. The sensitization of D1 receptor change the coupling efficiency of corresponding $\mathrm{G}$ proteins, activation of downstream intracellular signaling molecules, which lead the abnormal gene expression and protein synthesis ${ }^{[11]}$. Moreover, studies have found that the activation of the dopamine D3 receptor can reduce the accumulation of $\alpha$-synuclein and regulate the intracellular signal transduction mediated by the D1 receptor to participate in the occurrence of dyskinesia's ${ }^{[12]}$. More and more evidences have proved that complex neurotransmitter networks play an important role in LID. Nondopaminergic neurotransmitters are recognized as being involved in modulation of the basal ganglia and other neural circuits important for movement, which include glutamatergic, 5-HT, adenosine, acetylcholine, adrenaline and cannabis histamines, etc. these additional neurotransmitters have emerged as promising targets for novel treatments. Abnormal activity of glutamate pathway appears to be a main factor in PD patients with LID $^{[13]}$. Glutamate is the main excitatory neurotransmitter of the central nervous system, among which the ionotropic receptor N-methyl-D-aspartate (NMDA) receptor, alpha-amino-3-hydroxy-5-methyl-4-isoxazole propionic acid (AMPA) receptor and metabotropic receptor (metabotropic glutamate receptor (mGluR)) are closely related to dyskinesia. Further studies have found that the abnormal phosphorylation of striatum NMDA receptor subunits, mainly involves ionotropic glutamate receptors (GluN2A) and (GluN2B) subunits, 
which enhance glutamatergic excitatory transmission and involve in the development of $\mathrm{LID}^{[14]}$. Amantadine is a classic low-affinity, non-competitive NMDA antagonist. ADS-5102, amantadine sustained-release capsule, has shown obvious anti-dyskinesia effects and better tolerance in clinical trials ${ }^{[15]}$. In addition, it was found that IEM 1460, the AMPA receptor antagonist, alleviated the symptoms of dyskinesia in animal models, suggesting that selective splicing of AMPA receptor subunits leads to the change of striatal plasticity and promotes the occurrence of $\operatorname{LID}^{[16]}$. Moreover, activation of metabotropic glutamate receptor 5 (mGlu5) receptors can enhance NMDA receptor signal transduction and interact with D1/D5 DA receptors to jointly activate downstream signaling pathways ${ }^{[17]}$. AFQ056, a negative allosteric regulator of mGlu5 receptor, significantly reduced the abnormal involuntary movement scale (AIMS) compared with the placebo group and did not worsen the motor symptoms in a 13 $\mathrm{w}$ double-blind controlled trial ${ }^{[18]}$. As mentioned above, serotonergic neurons can produce and release DA when degeneration of nigrostriatal neurons occurs. However, DA is released in a non-physiological and uncontrolled manner, causing irregular stimulation of striatal DA receptors ${ }^{[7]}$.This kind of "false transmitter" has been widely studied for the drug intervention of 5-HT. F13714 and F15599, two highly selective "bias agonists" of 5-HT1A receptor, can reduce the 5-HT level in the striatum on both sides of the rat brain without worsening the Parkinson's symptoms. It is speculated that 5-HT1A receptor can weaken the peak of DA level and mediate the anti-dyskinesia effect, which provides a new idea for the treatment of peak dose dyskinesia ${ }^{[19]}$. It has been further discovered that adenosine receptors are mainly involved in the regulation of dyskinesia. Studies have shown that A2A receptors are highly expressed on (gamma-aminobutyric acid (GABA)) GABAergic neurons and interact with dopamine D2, mGluR5 and NMDA receptors to inhibit D2 receptor-mediated signaling pathways to regulate motor behavior ${ }^{[20]}$. Istradefylline, $\mathrm{A} 2 \mathrm{~A}$ receptor antagonist, can reduce the "off time" of patients with dyskinesia's, however its efficacy against dyskinesia is still controversial. It is worth noting that it can be effective in combination with low-dose L-DOPA to improve PD motor symptoms ${ }^{[21]}$. A cholinergic system is a significant factor in mediating striatal function. They modulate striatal output via muscarinic acetylcholine receptors (mAChRs) and nicotinic acetylcholine receptors (nAChRs). MAChRs directly regulate the activity of spinous neurons through M1 and M4 receptors and participate in the expression of dyskinesia. Activation of M1 receptors depresses $\mathrm{K}^{+}$ currents and enhances $\mathrm{Na}^{+}$currents, which increases MSN excitability. By contrast, M4 receptor decreases the excitability of spinous neurons and promotes the formation of LTD. Enhanced cholinergic system is considered to be a main factor in the development of LID $^{[22]}$. A number of studies have found that the activation of nAChRs is the main contributor to striatal DA release. In addition, increasing evidence has confirmed that $\beta 2 * \mathrm{nAChRs}$ and $\alpha 7 \mathrm{nAChRs}$ play a major role in functional activity. On the one hand, $\beta 2 *$ nAChRs are highly expressed in dopaminergic terminals, on the other hand, $\alpha 7 \mathrm{nAChRs}$ is preferentially expressed on glutamatergic afferents. Therefore, nAChRs not only regulate the release of DA from striatum, but also indirectly regulate the activity of MSN to induce the formation of dyskinesia ${ }^{[23]}$. Nicotine is a kind of nonselective nAChR agonist, which shows obvious anti-dyskinesia effect in rodents and primates with dyskinesia. It is speculated that nicotine reduces LID via $\beta 2 *$ and $\alpha 7 \mathrm{nAChRs}^{[24]}$. However, the direct mechanism between cholinergic receptors and LID is still unclear, which needs further experimental study. Anatomically, the adrenergic system originates from locus coeruleus. It directly stimulates the substantia nigra striatum and affects the release of DA in the basal ganglia. In particular, $\alpha 2$ receptors are highly expressed in GABA neurons, regulating GABA release and activating direct pathways ${ }^{[25]}$. Fipamezole, a clinical potential of selective $\alpha 2$-adrenergic receptor antagonist, can reduce LID of primates with 1-methyl-4-phenyl1,2,3,6-tetrahydropyridine (MPTP) lesions without affecting its anti-Parkinson's disease effect. Fipamezole (90 mg) significantly reduced dyskinesia in US subjects in a subgroup specific analysis in a multicenter, doubleblind, randomized clinical study in the United States and India. In addition, fipamezole can cause transient elevated blood pressure and its adverse reactions are acceptable ${ }^{[26]}$. In addition to the above mechanisms, non-dopamine systems such as cannabinoid receptors, histamines and opioids are also involved in the formation of LID. Cannabinoid can directly (through cannabinoid receptor on nigrostriatal neurons) and indirectly (by regulating GABA and glutamate release) regulate DA transmission in basal ganglia ${ }^{[27]}$. Preclinical experiments have shown that the cannabinoid type 1 receptor $\left(\mathrm{CB}_{1}\right)$ may be a potential approach for treatment of LID. It is speculated that the activation of the $\mathrm{CB}_{1}$ reduces the release of glutamate and inhibits the direct pathway to produce anti-dyskinesia effects ${ }^{[28]}$. 
Histamine receptor $\mathrm{H}_{2}$ is highly expressed in striatum pallidum and substantia nigra, which can up-regulate the excitability of cholinergic system and participate in the regulation of $\mathrm{LID}^{[29]}$. Ramotidine, a clinically available histamine receptor $\mathrm{H}_{2}$ antagonist, has shown anti-dyskinesia properties in rodent models, however its efficacy has not been proven in clinical trials ${ }^{[30]}$. The opioid peptides and opioid receptors have been linked to the development of LID. Mu-opioid receptor antagonist (ADL5510), can reduce LID in primates at low and moderate doses but failure in higher dose due to loss of selectivity ${ }^{[31]}$. On the other hand, the activation of $\mu$ and $\delta$ receptors can change normal activation extracellular signal regulated kinase (ERK1/2) and participate in the development of dyskinesia ${ }^{[32]}$. The pathophysiological mechanism of LID is complex and still remains unclear. Nigrostriatal degeneration and chronic L-DOPA administration plays the basis role of dyskinesia's. Recently, it has been recognized that nondopaminergic systems play an important role of LID. Positive results of non-dopaminergic drugs have been founded by large number of pre-clinical studies. However, these results have not yet been translated into clinical trials. On one hand, the reason of failure may be the poor tolerance and side effects shown in the drug clinical trials, on the other hand, it may be caused by the difference between the animal model and the clinical reality. With the continuous in-depth study of the pathophysiological mechanism of LID, more precise and individualized treatments can be provided for LID in the future.

\section{Acknowledgements:}

his work was supported by The Second Affiliated Hospital of Fujian Medical University.

\section{Conflicts of interest:}

The authors report no conflicts of interest.

\section{REFERENCES}

1. Fahn S. The spectrum of levodopa-induced dyskinesias. Ann Neurol 2000.

2. Jankovic J. Motor fluctuations and dyskinesias in Parkinson's disease: clinical manifestations. Mov Disord 2005;20:S11-6.

3. Jenner P, McCreary AC, Scheller DK. Continuous drug delivery in early-and late-stage Parkinson's disease as a strategy for avoiding dyskinesia induction and expression. $\mathrm{J}$ Neural Transm 2011;118(12):1691-702.

4. Paille V, Brachet P, Damier P. Role of nigral lesion in the genesis of dyskinesias in a rat model of Parkinson's disease. Neuroreport 2004;15(3):561-4.

5. Jenner P. Wearing off, dyskinesia and the use of continuous drug delivery in Parkinson's disease. Neurol Clin 2013;31(3):S1735 .
6. Nicoletti A, Mostile G, Nicoletti G, Arabia G, Iliceto G, Lamberti $\mathrm{P}$, et al. Clinical phenotype and risk of levodopainduced dyskinesia in Parkinson's disease. J Neurol 2016;263(5):888-94.

7. Grosch J, Winkler J, Kohl Z. Early degeneration of both dopaminergic and serotonergic axons-a common mechanism in Parkinson's disease. Front Cell Neurosci 2016;10:293.

8. Cenci MA. Presynaptic mechanisms of L-DOPA-induced dyskinesia: the findings, the debate and the therapeutic implications. Front Neurol 2014;5:242.

9. Picconi B, De Leonibus E, Calabresi P. Synaptic plasticity and levodopa-induced dyskinesia: electrophysiological and structural abnormalities. J Neural Transm 2018;125(8):126371.

10. Nishijima H, Ueno T, Funamizu Y, Ueno S, Tomiyama M. Levodopa treatment and dendritic spine pathology. Mov Disord 2018;33(6):877-88.

11. Perez XA, Zhang D, Bordia T, Quik M. Striatal D1 medium spiny neuron activation induces dyskinesias in parkinsonian mice. Mov Disord 2017;32(4):538-48.

12. Solis $\mathrm{O}$, Garcia-Montes JR, Gonzalez-Granillo $\mathrm{A}, \mathrm{Xu} \mathrm{M}$, Moratalla R. Dopamine D3 receptor modulates L-DOPAinduced dyskinesia by targeting D1 receptor-mediated striatal signaling. Cereb Cortex 2017;27(1):435-46.

13. Mellone M, Gardoni F. Glutamatergic mechanisms in L-DOPA-induced dyskinesia and therapeutic implications. J Neural Transm 2018;125(8):1225-36.

14. Mellone M, Stanic J, Hernandez LF, Iglesias E, Zianni E, Longhi A, et al. NMDA receptor GluN2A/GluN2B subunit ratio as synaptic trait of levodopa-induced dyskinesias: from experimental models to patients. Front Cell Neurosci 2015;9:245.

15. Oertel W, Eggert K, Pahwa R, Tanner CM, Hauser RA, Trenkwalder C, et al. Randomized, placebo-controlled trial of ADS-5102 (amantadine) extended-release capsules for levodopa-induced dyskinesia in Parkinson's disease (EASE LID 3). Mov Disord 2017;32(12):1701-9.

16. Kobylecki C, Crossman AR, Ravenscroft P. Alternative splicing of AMPA receptor subunits in the 6-OHDA-lesioned rat model of Parkinson's disease and L-DOPA-induced dyskinesia. Exp Neurol 2013;247:476-84.

17. Pourmirbabaei S, Dolatshahi M, Rahmani F. Pathophysiological clues to therapeutic applications of glutamate mGlu5 receptor antagonists in levodopa-induced dyskinesia. Eur J Pharmacol 2019;855:149-59.

18. Stocchi F, Rascol O, Destee A, Hattori N, Hauser RA, Lang AE, Poewe W, Stacy M, Tolosa E, Gao H, Nagel J. AFQ056 in Parkinson patients with levodopa-induced dyskinesia: 13-week, randomized, dose-finding study. Mov Disord 2013;28(13):1838-46.

19. Newman-Tancredi A, Varney MA, McCreary AC. Effects of the serotonin 5-HT 1A receptor biased agonists, F13714 and F15599, on striatal neurotransmitter levels following 1-DOPA administration in hemi-Parkinsonian rats. Neurochem Res 2018;43(5):1035-46.

20. Pinna A, Serra M, Morelli M, Simola N. Role of adenosine A 2A receptors in motor control: relevance to Parkinson's disease and dyskinesia. J Neural Transm 2018;125(8):1273-86.

21. Tomiyama M. Adenosine receptors and dyskinesia in pathophysiology. Int Rev Neurobiol 2014;119:117-26.

22. Perez XA, Bordia T, Quik M. The striatal cholinergic system in L-dopa-induced dyskinesias. J Neural Transm 2018;125(8):1251-62. 
23. Quik M, Wonnacott S. $\alpha 6 \beta 2 *$ and $\alpha 4 \beta 2 *$ nicotinic acetylcholine receptors as drug targets for Parkinson's disease. Pharmacol Rev 2011;63(4):938-66.

24. Quik M, Mallela A, Ly J, Zhang D. Nicotine reduces established levodopa-induced dyskinesias in a monkey model of Parkinson's disease. Mov Disord 2013;28(10):1398-406.

25. Ostock CY, Hallmark J, Palumbo N, Bhide N, Conti M, George JA, et al. Modulation of L-DOPA's antiparkinsonian and dyskinetic effects by $\alpha 2$-noradrenergic receptors within the locus coeruleus. Neuropharmacology 2015;95:215-25.

26. LeWitt PA, Hauser RA, Lu M, Nicholas AP, Weiner W, Coppard N, et al. Randomized clinical trial of fipamezole for dyskinesia in Parkinson disease (FJORD study). Neurology 2012;79(2):163-9.

27. Garcia C, Palomo-Garo C, Gomez-Galvez Y, Fernandez-Ruiz J. Cannabinoid-dopamine interactions in the physiology and physiopathology of the basal ganglia. Br J Pharmacol 2016;173(13):2069-79.

28. Junior NC, dos-Santos-Pereira M, Guimaraes FS, Del Bel E. Cannabidiol and cannabinoid compounds as potential strategies for treating Parkinson's disease and L-DOPAinduced dyskinesia. Neurotox Res 2020;37(1):12-29.

29. Lim SA, Xia R, Ding Y, Won L, Ray WJ, Hitchcock SA, et al. Enhanced histamine $\mathrm{H}_{2}$ excitation of striatal cholinergic interneurons in L-DOPA-induced dyskinesia. Neurobiol Dis 2015;76:67-76.
30. Mestre TA, Shah BB, Connolly BS, de Aquino C, Al Dhakeel $\mathrm{A}$, Walsh $\mathrm{R}$, et al. Famotidine, a Histamine $\mathrm{H}_{2}$ Receptor Antagonist, Does Not Reduce Levodopa-Induced Dyskinesia in Parkinson's Disease: A Proof-of-Concept Study. Mov Disord Clin Pract 2014;1(3):219-24.

31. Koprich JB, Fox SH, Johnston TH, Goodman A, Le Bourdonnec $\mathrm{B}$, Dolle RE, et al. The selective mu-opioid receptor antagonist ad15510 reduces levodopa-induced dyskinesia without affecting antiparkinsonian action in mptp-lesioned macaque model of Parkinson's disease. Mov Disord 2011;26(7):122533.

32. Pan J, Cai H. Opioid system in L-DOPA-induced dyskinesia. Transl Neurodegener 2017;6(1):1-5.

This is an open access article distributed under the terms of the Creative Commons Attribution-NonCommercial-ShareAlike 3.0 License, which allows others to remix, tweak, and build upon the work non-commercially, as long as the author is credited and the new creations are licensed under the identical terms

This article was originally published in a special issue,

"Therapeutic Perspectives in Biomedical Research and Pharmaceutical Sciences and their Nursing Methods"

Indian J Pharm Sci 2021:83(4)Spl issue "139-143" 